\title{
The V-val subtype Epstein-Barr virus nuclear antigen 1 promotes cell survival after serum withdrawal
}

\author{
MEI CHAO ${ }^{1,2}$, HSIAO-NING WANG ${ }^{1}$, YI JIN LU ${ }^{1}$, YU-SUN CHANG ${ }^{1,3}$ and JAU-SONG YU ${ }^{3,4}$ \\ ${ }^{1}$ Division of Microbiology, Graduate Institute of Biomedical Sciences, ${ }^{2}$ Department of Microbiology and \\ Immunology, ${ }^{3}$ Molecular Medicine Research Center, College of Medicine, ${ }^{4}$ Department of Biochemistry \\ and Molecular Biology, Chang Gung University, Kwei-Shan, Tao-Yang 333, Taiwan R.O.C.
}

Received August 7, 2014; Accepted November 3, 2014

DOI: $10.3892 /$ or.2014.3625

\begin{abstract}
Epstein-Barr virus (EBV) can establish latent infection and has been associated with various human cancers. Epstein-Barr nuclear antigen 1 (EBNA1) is the only viral protein that is expressed in all EBV-associated malignant tissues. The $\mathrm{N}$ - and C-terminal domains of EBNA1, which are connected by internal glycine/alanine-rich short repeat sequences of various sizes, show sequence divergence across EBV strains isolated from around the world. At least five subtypes have been described, according to the amino acid at residue 487: P-ala, P-thr, V-val, V-pro, and V-leu. Whether the variations of EBNA-1 contribute to the pathogenesis of EBV or simply reflect the geographical distribution of EBV remain to be investigated. Furthermore, the cell effects conferred by EBNA1 subtypes that differ from that of the B95.8 prototype, which belongs to the P-ala subtype, remain to be elucidated. In this study, PCR was amplified with the full-length $\mathrm{V}$-val EBNA1 gene from the CG3 cell line, an EBV-carrying lymphoblastoid cell line derived from a Taiwanese chronic myeloid leukemia patient. Plasmids expressing His-tagged EBNA1 fusion proteins in $E$. coli were constructed and used to raise antibodies in rabbit. The V-val EBNAl gene was then cloned into a eukaryotic expression vector and successfully expressed in the transfected cultured cells. Expression of V-val EBNA1 rendered 293 cells able to undergo serum-independent cell proliferation, providing them with anti-apoptotic abilities, which are two characteristics of cancer cells. These data suggested that use of EBNA1 originally derived from tumor cells, rather than the more commonly utilized prototype, when investigating the potential role of EBNA1 in the oncogenesis of EBV-associated malignancies, is crucial.
\end{abstract}

Correspondence to: Professor Mei Chao, Department of Microbiology and Immunology, College of Medicine, Chang Gung University, 259 Wen-Hwa 1st Road, Kwei-Shan, Tao-Yang 333, Taiwan, R.O.C. E-mail: pa0728@mail.cgu.edu.tw

Key words: Epstein-Barr virus, Epstein-Barr nuclear antigen 1, $\mathrm{V}$-val subtype, cell proliferation, apoptosis

\section{Introduction}

Epstein-Barr virus (EBV), a ubiquitous human $\gamma$ herpes virus, establishes persistent latent infections and has been causally associated with a variety of B-cell and epithelial cell malignancies, including Burkitt's lymphoma (BL), Hodgkin's lymphoma, gastric carcinoma and nasopharyngeal carcinoma (NPC) (1). EBV depends on Epstein-Barr nuclear antigen 1 (EBNA1) to mediate critical processes in the viral life cycle, and EBNA1 is the only viral protein that has been identified in all EBV-associated malignant tissues. It is wellknown that EBNA1 expression is necessary for maintaining EBV in latently proliferating cells, mediating synthesis of the EBV genome, non-randomly partitioning the virus to daughter cells (2-4) and regulating viral gene transcription (5-9). The effects of EBNA1 on the host cell remain to be elucidated $(10,11)$, although it has been suggested that EBNA1: is critical for the continued survival of BL (12), may affect the function of p53 by competing for the USP7 ubiquitin-specific protease $(13,14)$, promotes the development of B-cell lymphoma in transgenic mice (15), counteracts Nm23-H1-mediated suppression of cell migration $(16,17)$, serves as a potential regulator of signaling pathways (18-21), and increases reactive oxygen species levels and genomic instability in BL cell lines $(22,23)$. The aforementioned findings suggest that EBNA1 may play a direct role in promoting the tumorigenesis of EBV-associated malignancies.

The molecular weight of EBNA1 varies from 69 to $96 \mathrm{kDa}$, depending on the number of internal glycinealanine repeats (24-26), which contribute to downregulating EBV-mediated immune responses by inhibiting antigen presentation (27). Sequence variations in EBNA1 relative to that of the prototypic B95.8 strain were first described in sequences $\mathrm{N}$ - and $\mathrm{C}$-terminal to the internal repeat regions of EBNA1 proteins isolated from EBV-positive NPC samples obtained in Hong Kong (28). Subsequently, numerous EBNA1 sequences have been identified from healthy individuals and patients with various EBV-associated diseases in different geographic regions (29). Gutiérrez et al (30) classified EBNA1 into the P-ala, P-thr, V-val, V-leu and V-pro subtypes, which were termed according to the amino acid (aa) at position 487 in EBNA1 (numbered according to prototype B95.8 strain). Sub-variants of P-ala, P-thr and V-val, have also been characterized (31-33). Previously, it was shown that within a given 
Table I. List of primers used for PCR amplification of EBNA 1 sequences.

\begin{tabular}{llc}
\hline Primers & \multicolumn{1}{c}{ Sequence (5'-3') } & B95.8 coordinates \\
\hline F1 & CGTCCAGCAAAAAGGGGGACGAG & $107671-107693$ \\
F2 & GCGGAGCTGAGTGACGTGACAAC & $110062-110040$ \\
GA1 & CCATGGACGAGGACGGGGAAG & $108063-108083$ \\
GA2 & GCTTGGGCCTTCTCCTGGGTCAT & $109290-109266$ \\
GA3 & AGGACGAGGAGGCGGAAGACCAG & $108090-108112$ \\
GA4 & TGCGGGGCCCTGCTCTATCG & $109266-109247$ \\
R1 & CGCCTAGCATATGATGTCTGACGAGGGGCCA & NdeI-107950-107967 \\
R2 & CCGGGATCCTTATCCTGTTCCACCGTGGGTC & BamHI-108219-108202 \\
R3 & CGCCTAGCATATGAGGCGCAAAAAAGGAGGGTGG & NdeI-109323-109344 \\
R4 & CCGGGATCCTTAATATACGAACACACCGGCGAC & BamHI-109875-109855 \\
R5 & CGCCTAGCATATGCGGGGTAGAGGACGTGAAAGA & NdeI-109009-109029 \\
\hline
\end{tabular}

geographic area, tumor-associated EBNA1 subtypes were also prevalent in the general population (34). For example, V-val EBNA1 was found exclusively in southeastern Chinese NPC samples, and was also the predominant subtype observed in this general population $(32,33,35-37)$. However, most of the molecular studies on the biological functions of full-length EBNA1 have used the sequence from prototype B95.8, a strain originating from an infectious mononucleosis patient in Africa, and whose EBNA1 belongs to the P-ala subtype (30). Thus it is important to investigate whether any functional differences are associated with genetic variations in EBNA1, and the manner in which they affect risk of disease. Results of such an investigation may provide a better understanding of the molecular mechanisms underlying the associations between EBV-related disease and particular cell types and/or genetic backgrounds.

Consequently, we cloned the full-length EBNAl gene from CG3 cells (38). This EBV-containing lymphoblastoid cell line was originally established from the bone marrow cells of a chronic myeloid leukemic patient from Taiwan, with V-val being the predominant EBNA1 subtype. As expected, the EBNA1 detected in CG3 cells belonged to the V-val subtype. Subsequently, we purified His-tagged, bacterially expressed $\mathrm{N}$ - and C-terminal CG3 EBNA1 fusion proteins and used them to raise EBNA1-reactive antibodies in rabbits. Additionally, we successfully expressed the full-length CG3 EBNA1 in eukaryotic cells, generating an experimental system that was subsequently used to elucidate the oncogenic potential of this V-val EBNA1 subtype.

\section{Materials and methods}

Cell lines. CG3 (38) is a spontaneous EBV-carrying lymphoblastoid cell line that was derived from a Taiwanese patient with juvenile chronic myeloid leukemia (kindly provided by Dr Y.S. Chang). B95.8 (39) is a marmoset lymphoid cell line that was EBV-immortalized with a virus originating from an infectious mononucleosis patient, sampled in Africa. Raji (40), P3HR1 (41) and Daudi (42) are EBV-containing human lymphoid cell lines harboring viruses from Burkitt's lymphoma patients. The cells were maintained in RPMI-1640 supplemented with $10 \%$ fetal calf serum (FCS), at $37^{\circ} \mathrm{C}$ in a humidified $5 \% \mathrm{CO}_{2}$ atmosphere. The 293 cell line (a human embryonal kidney cell line) (43) was grown in DMEM supplemented with $10 \%$ FCS. The 293-EBNA cells (i.e., 293 cells stably expressing B95.8 EBNA1; Invitrogen-Life Technologies, Carlsbad, CA, USA) were maintained in DMEM-10\% FCS containing $250 \mu \mathrm{g} / \mathrm{ml}$ of Geneticin (G418; Gibco-Life Technologies, Carlsbad, CA, USA).

PCR amplification and sequence analysis of the EBNA1 gene from cultured cells. DNA was extracted from CG3 and 293-EBNA cells using a QIAamp DNA Mini kit (Qiagen, Valencia, CA, USA) according to the manufacturer's instructions. The full-length $E B N A 1$ gene and the sequence encoding its middle repeat region were PCR-amplified using the GC-RICH PCR System (Roche Molecular Biochemicals) according to the manufacturer's instructions. The sequences of the utilized primers are shown in Table I, with the nucleotide numbering system used in this report being in accordance with that of Baer et al (44). For all PCR reactions discussed herein, the input DNAs were preheated at $95^{\circ} \mathrm{C}$ for $5 \mathrm{~min}$ prior to cycling, and the PCR products were subjected to a final extension for $10 \mathrm{~min}$ at $72^{\circ} \mathrm{C}$. Briefly, $100 \mathrm{ng}$ of DNA was combined with the provided reaction mixture (containing $0.5 \mathrm{M}$ GC-RICH resolution solution) and subjected to PCR. To amplify the full-length EBNA1 gene, the F1/F2 primers were used along with the following reaction conditions: 30 cycles of $95^{\circ} \mathrm{C}$ for $1 \mathrm{~min}, 63^{\circ} \mathrm{C}$ for $2 \mathrm{~min} 30 \mathrm{sec}$ and $72^{\circ} \mathrm{C}$ for $1 \mathrm{~min}$. Nested PCR was required to successfully amplify the internal repeat sequences of EBNA1 (Fig. 1). Primer pairs GA1/GA2 and GA3/GA4 were used for the first and second round of PCR, respectively, under the aforementioned reaction conditions. The PCR products representing the full-length EBNAl gene from CG3 and 293-EBNA cells were purified and cloned into a T-vector (pCRII-TOPO; Invitrogen) and three independent clones were sequenced (ABI377 DNA sequencing system; Perkin-Elmer/Applied Biosystems). From the clone, plasmids containing consensus sequences for pCRII-TOPO-CG3.1 and pCRII-TOPO-B95.8.1 were selected and used to generate EBNA1 eukaryotic expression vectors that expressed the CG3 and B95.8 EBNA1s, respectively (see below). 


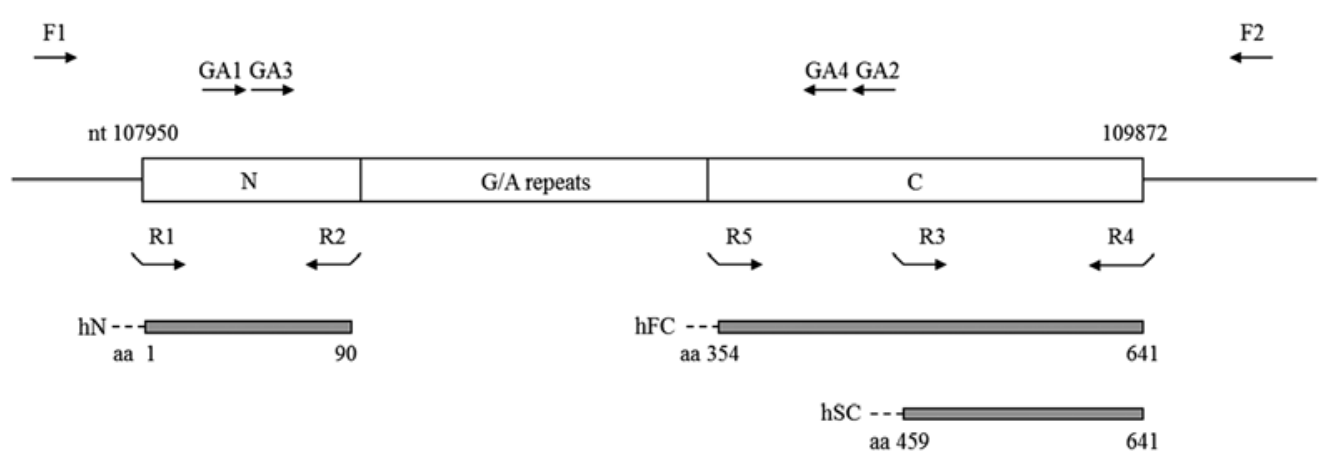

Figure 1. Strategy used to clone the EBNA1 gene. The structure of the EBNA1 gene (nt 107950-109872) is shown as the N-and C-terminal portions separated by the internal glycine/alanine repeat sequence. The positions of the utilized PCR primers are indicated by arrows. The bottom diagram summarizes the regions covered by the three His-tagged EBNA1 fusion proteins, which are indicated as hN (aa 1-90), hFC (aa 354-641) and hSC (aa 459-641). The His-tag is denoted by the dotted lines.

Expression and purification of recombinant EBNAl proteins in E. coli. pCRII-TOPO-CG3.1 served as the template for PCR amplifying three fragments: the $\mathrm{N}$ fragment covering the N-terminus of EBNA1 (aa 1-90), and fragments FC and $\mathrm{SC}$ corresponding to two C-terminal regions downstream of the glycine-alanine repeat region (aa 354-641 and 459-641, respectively) (Fig. 1). The N, SC and FC fragments were amplified with primer pairs R1/R2, R3/R4 and R5/R4 (Table I and Fig. 1), respectively. For cloning purposes, restriction enzyme sites were included in the primers. The PCR products were then subjected to NdeI and BamHI double digestion followed by gel purification. The purified products were inserted in-frame at the C-terminus of the His-tag in pET-15b plasmids (Novagen) which had been pretreated with $N d e$ I and $B a m \mathrm{HI}$. The resulting plasmids containing the N, FC and $\mathrm{SC}$ inserts were designated as $\mathrm{pET}_{15 \mathrm{~b}}-\mathrm{hN}, \mathrm{pET}_{15 \mathrm{~b}}-\mathrm{hFC}$ and $\mathrm{pET}_{15 \mathrm{~b}}-\mathrm{hSC}$, respectively, and their encoded fusion proteins were referred to as $\mathrm{hN}, \mathrm{hFC}$ and $\mathrm{hSC}$, respectively. These plasmids were used to transform BL21(DE3)pLysS (Novagen), and the fusion proteins were induced and purified as per the manufacturer's instructions. Briefly, bacteria were grown to an $\mathrm{OD}_{600}$ of $0.6,1 \mathrm{mM}$ IPTG was added, and the incubation was continued for $3 \mathrm{~h}$ via agitation. The bacterial pellet was collected by centrifugation at $2500 \mathrm{~g}$ for $15 \mathrm{~min}$ and the cells were lysed by freeze-thawing. The soluble recombinant EBNA1 proteins were purified with one-step metal chelation chromatography (Novagen). The purified hFC was then concentrated using Ultracel-3K (Millipore, Billerica, MA, USA). Equal amounts of the purified fractions were collected, resolved by SDS-PAGE and subjected to Coomassie blue staining. Western blot analysis, as previously described (45), was performed using an anti-His antibody (diluted 1:1,000; Santa Cruz Biotechnology, Inc., Santa Cruz, CA, USA).

Immunization of animals. The backs of New Zealand White rabbits were subcutaneously immunized with $300 \mu \mathrm{g}$ of $\mathrm{hN}$ or hSC in $0.5 \mathrm{ml}$ complete Freund's adjuvant (v/v 1:1) on day 0, and with the same amount of the corresponding protein in incomplete Freund's adjuvant (v/v 1:1) (Sigma-Aldrich) on day 20 and were bled on day 27. The obtained antisera were designated $\mathrm{Ab}-\mathrm{N}$ and $\mathrm{Ab}-\mathrm{SC}$, respectively. The animal experimental protocol was reviewed and approved by the Chang Gung University Animal Ethics Committee. The antibody responses were examined by western blot analysis using purified recombinant proteins and extracts prepared from EBV-containing cell lines and EBNA1-transfected 293 cells.

Expression of EBNA1 from eukaryotic vectors. pCRII-TOPOCG3.1 and pCRII-TOPO-B95.8.1 were digested with BamHI, filled in with Klenow (New England Biolabs, Beverly, MA, USA), and cleaved with $X b a$ I to release the inserts of interest. Plasmid pCMV-EBNA (Clontech), which encoded EBNA1 of the B95.8 strain, was treated with PstI, filled in with Klenow, and digested with $\mathrm{XbaI}$ to remove the B95.8 EBNAl gene, which served as the vector. The insert and vector were gel purified and ligated to generate the eukaryotic expression plasmids, pCMV-CG3EBNA1 and pCMV-B95.8EBNA1. These plasmids were transfected into 293 cells. Briefly, 5x10 cells/wel) were seeded in 6-well (35-mm diameter) plates, grown for $18 \mathrm{~h}(\sim 60-70 \%$ confluency), and then transfected with $4 \mu \mathrm{g}$ DNA and $10 \mu \mathrm{l}$ Lipofectamine (Invitrogen), according to the manufacturer's instructions. At $48 \mathrm{~h}$ post-transfection, the cell extracts were collected, resolved by $10 \%$ SDS-PAGE, and subjected to western blot analysis (45) with rabbit Ab-SC antibodies. Proteins extracted from 293-EBNA (Invitrogen) and CG3 cells were used as positive controls. To generate 293 cells stably expressing EBNA1 proteins of B95.8 or CG3 origin, or the drug-resistant gene alone (as a control), EBNA1 expression vector- and empty vector-transfected cells were selected in the presence of $500 \mu \mathrm{g} / \mathrm{ml}$ Geneticin (G418; Gibco) and then maintained in medium containing $250 \mu \mathrm{g} / \mathrm{ml}$ Geneticin.

Serum withdrawal and cell survival. Stably transfected 293 cells $\left(2 \times 10^{5}\right.$ cells/well) were seeded in 6 -well plates. At $16 \mathrm{~h}$ post-seeding, the cells were washed twice with serum-free medium and then cultured in the absence and presence of FCS. Cell viability was determined at various time points using trypan blue exclusion. Proteins were also extracted at different time points and subjected to western blot analysis using antibodies against poly(ADP-ribose) polymerase (PARP) and actin (diluted 1:600 and 1:5,000, respectively; both from Millipore).

\section{Results}

The EBNA1 sequence in CG3 cells. Size polymorphisms and sequence variations have been reported in EBNA1 proteins 

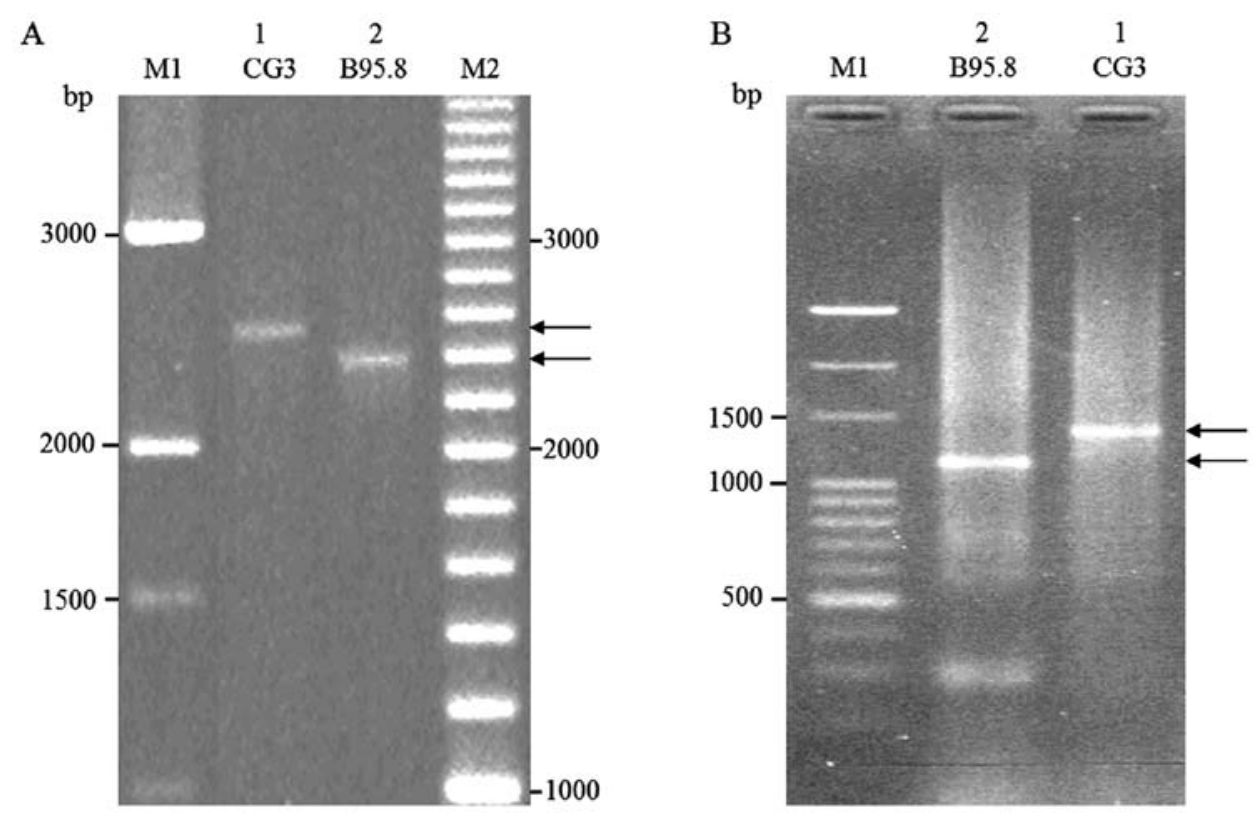

Figure 2. PCR-amplified products of (A) the full-length EBNA1 gene and (B) its middle repeat region, as resolved by 1 and $2 \%$ agarose gel electrophoresis, respectively, and visualized by ethidium bromide staining and UV transillumination. Lanes: 1, PCR products from CG3 cells; 2, PCR products from B95.8 cells; and M1 and M2, DNA size markers.

from different EBV isolates. CG3 is an EBV-containing lymphoblastoid cell line established from a Taiwanese patient with chronic myeloid leukemia (38). To the best of our knowledge, no previous study has reported the size and sequence of its EBNA1 protein. Consequently, PCR was amplified with the full-length EBNAl gene from CG3 cells. As shown in Fig. 1, PCR reactions using primers F1/F2 and nested primers GA1/GA2 and GA3/GA4 were performed to amplify the full-length EBNA1 gene and its middle domain, respectively. DNA extracted from B95.8 cells was amplified as a control. The full-length gene and its middle region from B95.8 cells were previously predicted to be 2392 and $1177 \mathrm{bp}$ in length, respectively (44). We obtained PCR products consistent with those predicted sizes (Fig. 2A and 2B, lane 2). The PCR products representing the full-length gene and the middle region of EBNA1 from CG3 cells (Fig. 2A and B, lane 1) appeared to be $\sim 150$ bp longer than those from B95.8 cells (Fig. 2A and B, lane 2). These data are consistent with previous studies that the molecular mass polymorphisms of EBNA1 across different EBV isolates reflect different numbers of internal glycinealanine repeats (24-26).

Sequence analysis revealed that the $\mathrm{N}$ - and C-terminal regions of the CG3 EBNAl gene did not contain any insertion or deletion, but harbored a series of nucleotide changes relative to the B95.8 clone. The deduced amino acid sequences of the CG3 clone relative to the B95.8 EBNA1 sequence are shown in Fig. 3. Based on the amino acid at position 487 and the characterized sequence variations at other positions, we determined that the CG3 EBNA1 belongs to the V-val subtype. We identified 4 (aa 16, 18, 20 and 85) and 12 (aa 364, 410, 411, 418, $439,487,499,502,524,528,533$ and 594) amino acid substitutions relative to the $\mathrm{B} 95.8$ sequence in the $\mathrm{N}$ - and $\mathrm{C}$-terminal regions, respectively, of the CG3 EBNA1. By contrast, only three amino acid substitutions (aa 85, 364 and 410) were identified between the CG3 EBNA1 and the EBNA1 isolated from
NPC patients of Hong Kong (28), which was the first reported V-val EBNA1.

Isolation of EBNA1 fusion proteins and generation of rabbit anti-EBNA1 antibodies. Recombinant CG3 EBNA1 proteins covering aa 1-90, 354-641 and 459-641 were expressed as His-tagged $\mathrm{N}$-terminal fusion proteins in E. coli, and were designated as $\mathrm{hN}-$, hFC- and hSC-EBNA1, respectively (Fig. 1). SDS-PAGE and Coomassie blue staining were used to examine the EBNA1 fusion proteins before (Fig.4A-C, lane C) and after (lanes 1-4) their purification by metal chelation chromatography. The sizes of the EBNA1 fusions were as expected, except that doublet bands were observed for $\mathrm{hN}$ (Fig. 4A, lanes 2 and 3). The molecular nature of these doublets remains unknown. As shown in Fig. 4D, fraction 2 of $\mathrm{hN}$ (lane 1), fraction 3 of hSC (lane 2), and concentrated pooled hFC (fractions 2 and 3, lane 3) were stained with Coomassie blue. These purified EBNA1 fusions were then analyzed by western blotting using an anti-His antibody (Fig. 4E).

The purified $\mathrm{hN}$ and $\mathrm{hSC}$ fusion proteins were used to raise antibodies in rabbits, and the resulting antisera were designated as $\mathrm{Ab}-\mathrm{N}$ and $\mathrm{Ab}-\mathrm{SC}$, respectively. As expected, our western blot analysis showed that $\mathrm{Ab}-\mathrm{N}$ reacted with purified $\mathrm{hN}$ (although a significant background was observed; Fig. 5A), while $\mathrm{Ab}-\mathrm{SC}$ detected $\mathrm{hSC}$ and $\mathrm{hFC}$ but not hN (Fig. 5B). We determiend the ability of Ab-SC to detect EBNA1 from cell lines carrying various EBNA1 subtypes. As reported in the present and other studies $(46,47)$, the EBNA1 proteins in the B95.8, P3HR1, Daudi and CG3 cell lines correspond to the P-ala, V-leu, P-thr and V-val subtypes, respectively. The EBNA1 protein in Raji cells corresponds to the P-ala subtype, but has amino acid substitutions with respect to B95.8 EBNA1 (47). As shown in Fig. 5C, the Ab-SC antibody reacted with EBNA1 in all of the tested EBNA1-positive cells. As reported previously (26), the molecular weights of the EBNA1 


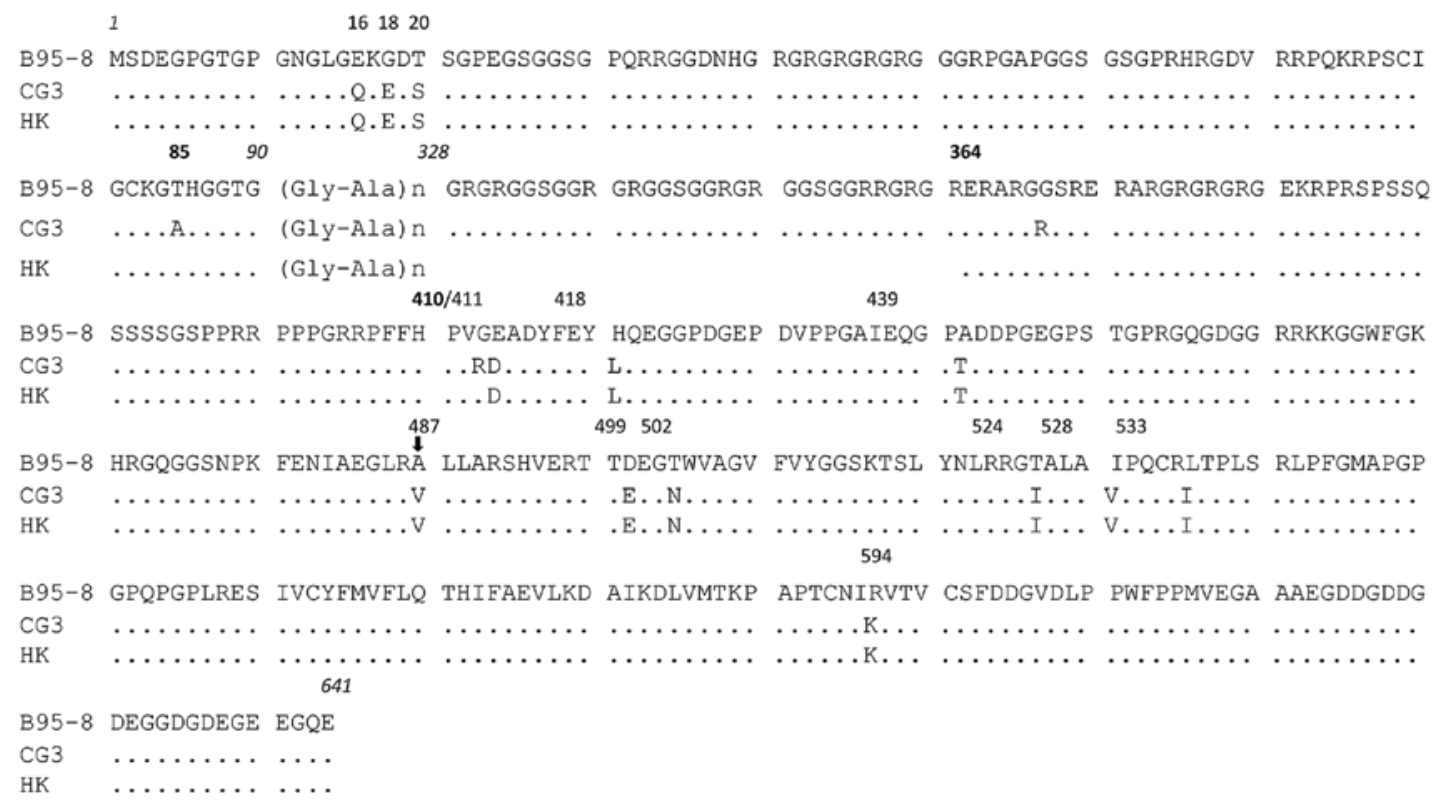

Figure 3. Amino acid alignment of the $\mathrm{N}$ - and C-terminal regions of CG3 EBNA1 relative to the prototype B95.8 EBNA1 sequence. The conserved amino acids are indicated with dots. 'HK' is the conserved sequence detected in NPC samples from Hong Kong (28). The position of aa 487 is indicated with an arrow.

A

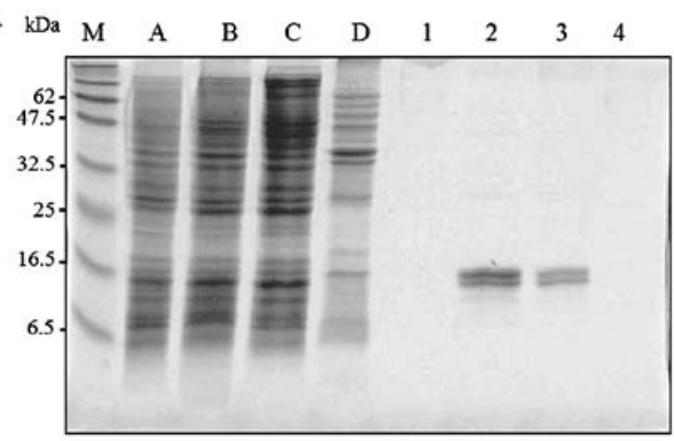

C

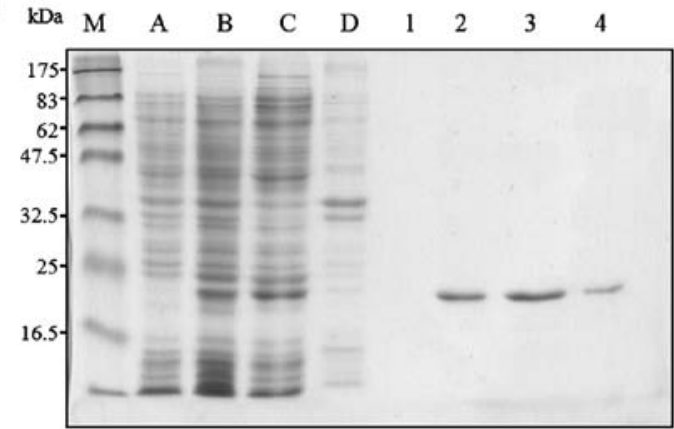

B

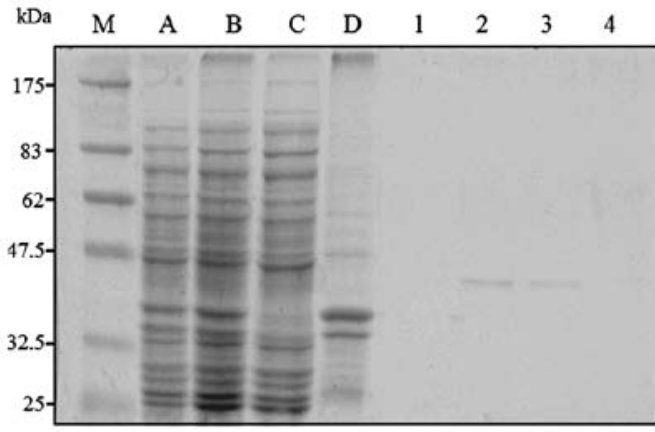

D $\quad \begin{array}{llll} & \mathrm{KDa} & 1 & 2\end{array}$

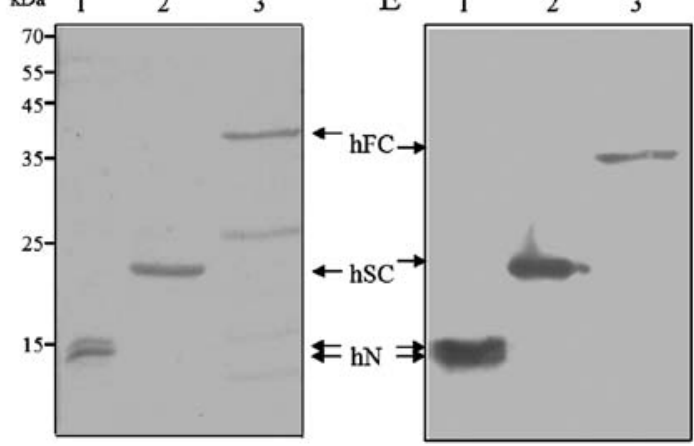

Figure 4. Expression and purification of recombinant EBNA1s expressed in E. coli. The His-tagged EBNA1 fusion proteins, (A) hN-, (B) hFC- and (C) hSC-EBNA1, were expressed in E. coli and recovered by affinity purification. The purified proteins were separated by 15,10 , and $12.5 \%$ SDS-PAGE, respectively, and stained with Coomassie blue. Lanes: A and B, protein extracts obtained before and after IPTG induction; C and D, soluble and pellet fractions after induction; and 1-4, the purified fractions. (D and E) The purified proteins were separated by $12 \%$ SDS-PAGE followed by (D) Coomassie blue staining or (E) western blot analysis using an anti-His antibody. Lanes: 1, hN-EBNA1; 2, hSC-EBNA1; and 3, hFC-EBNA1. The mobilities of prestained protein markers (GeneTeks BioScience Inc., Taipei, Taiwan) are indicated on the left.

proteins in Raji, B95.8, Daudi, and P3HR1 cells were 69, 79, 78 , and $75 \mathrm{kDa}$, respectively. We obtained results consistent with those prior findings (Fig. 5C), and found that the CG3 EBNA1 was larger than the B95.8 EBNA1 in our western blot analysis (similar to the gene-based findings described earlier and shown in Fig. 2). Collectively, these results showed that our rabbit polyclonal anti-EBNA1 antibodies detected EBNA1 fusion proteins expressed in E. coli, and EBNA1 proteins of various subtypes in different EBV-associated cell lines.

Cloning and expression of the full-length CG3 V-val EBNAI in cultured cells. Since most of the previous functional studies 

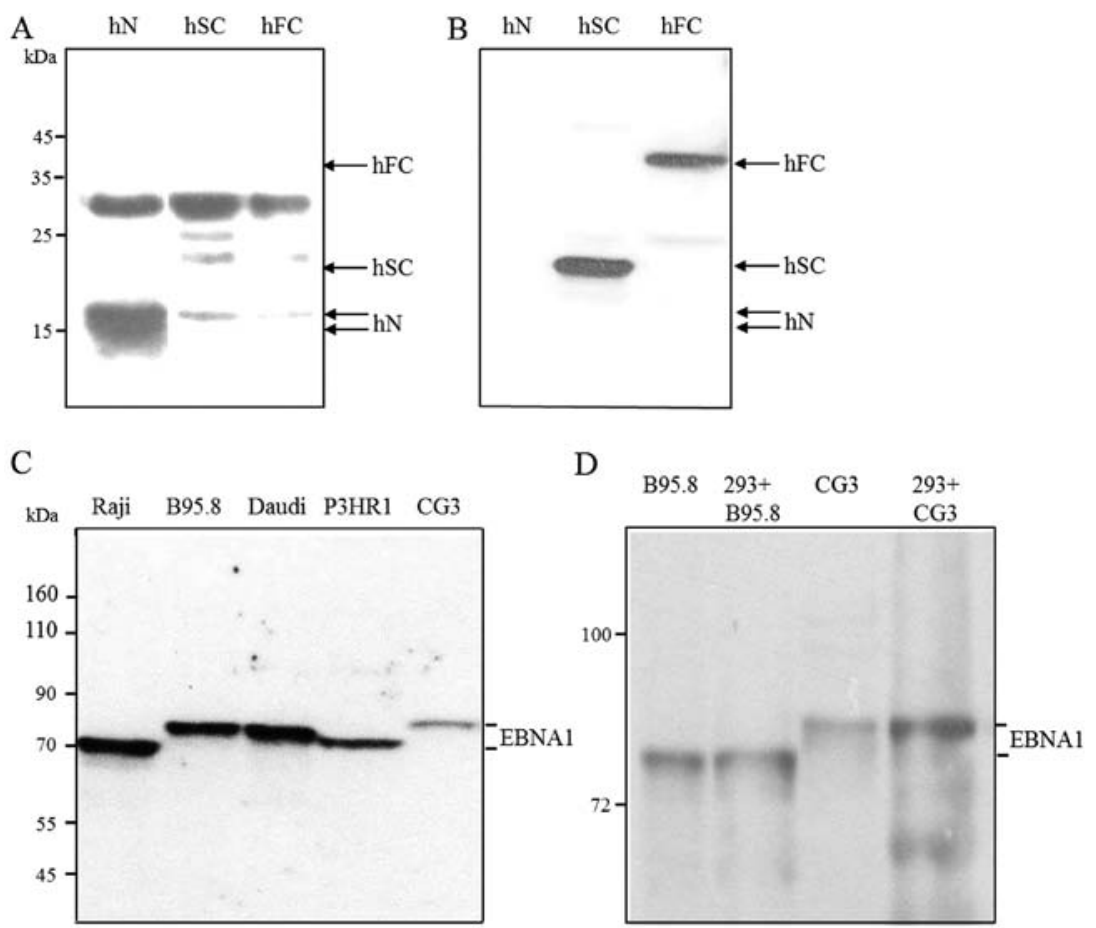

Figure 5. Characterization of rabbit antibodies raised against EBNA1. Purified His-tagged hN, hSC and hFC-EBNA1 fusion proteins were subjected to western blot analysis using our (A) Ab-N (diluted 1:1,000) and (B) Ab-SC (diluted 1:2,000) antibodies. The mobilities of the EBNA1 fusion proteins are shown on the right. (C) Western blot analysis of the reactivity of the rabbit polyclonal Ab-SC antibody to lysates prepared from different EBV-positive cells. Protein samples were separated by 10\% SDS-PAGE. (D) Expression of full-length V-val CG3 EBNA1 in transfected eukaryotic cells. EBNA1-expressing plasmids were used to transfect 293 cells. After $48 \mathrm{~h}$, the cell lysates were resolved by 10\% SDS-PAGE and the EBNA1 proteins were detected with our rabbit Ab-SC antibody Cell lysates prepared from CG3 and B95.8 cells were used as size controls. The mobilities of the various EBNA1s are shown on the right, and the mobilities of prestained protein markers (GeneTeks BioScience Inc.) are indicated on the left.

involved EBNA1 cloned from the B95.8 prototype (which belongs to the P-ala subtype), we subcloned the full-length V-val CG3 EBNA1 gene into a mammalian expression vector. The resulting plasmid, pCMV-CG3EBNA1, was transfected into 293 cells, and EBNA1 expression was examined by western blot analysis using our rabbit Ab-SC antibody (Fig. 5D). The electrophoretic mobility of the expressed CG3 EBNA1 was retarded as compared to that of B95.8 EBNA1, and the plasmid-expressed CG3 and B95.8 EBNA1 proteins were indistinguishable from those of the corresponding EBV-positive cells. These findings indicated that the full-length EBNA1 gene was successfully amplified from EBV-positive samples and expressed in the cultured cells, which in turn should facilitate studies on the functional differences among the different EBNA1 subtypes.

Expression of V-val CG3 EBNA1 supports serum-independent cell proliferation and blocks apoptosis. Growth factor-independent proliferation is considered to be a hallmark of cancer. To study the effect of CG3 EBNA1 on the cell proliferation and apoptosis of serum-starved cells, we generated 293 cells stably expressing CG3 EBNA1 (V-val subtype), B95.8 EBNA1 (P-ala subtype), or the drug-resistance gene alone (empty vector, control). Expression of CG3 EBNA1 (from two independent clones) and B95.8 EBNA1 was confirmed by western blot analysis using our Ab-SC antibody (Fig. 6A). The cells were then cultured in the absence of FCS, and cell viability was determined by trypan blue exclusion. As expected, 293 cell-expressing empty vector demonstrated loss of viability within three days of serum deprivation (Fig. 6B). Cells expressing B95.8 EBNA1 grew exponentially only for one day after serum withdrawal, and then plateaued through to the end of the experiment (day 5). As shown in Fig. 6B, however, the 293 cells expressing two independent clones of CG3 EBNA1 were able to proliferate under serum starvation throughout the experimental period. We also used western blotting to examine the status of PARP, a substrate cleaved by caspases during apoptosis, in 293 cells expressing empty vector, B95.8 EBNA1, and CG3 EBNA1 following serum withdrawal. As shown in Fig. 6C, cells expressing empty vector showed marginal induction of PARP cleavage after $63 \mathrm{~h}$ and significant PARP cleavage after $66 \mathrm{~h}$ of serum starvation. PARP cleavage was observed after $69 \mathrm{~h}$ of serum starvation in cells expressing B95.8 EBNA1. Of note, 293 cells expressing CG3 EBNA1 under serum starvation inhibited PARP cleavage at all of the tested time points. Taken together, these results suggested that CG3 EBNA1 blocked serum withdrawalinduced apoptosis and promoted cell proliferation.

\section{Discussion}

EBV has been associated with different malignancies in various geographic regions (1), and EBNA1, which is the only viral protein observed in all EBV-associated tumors, shows region-specific sequence variations $(29,34)$. The B85.8 EBNA1, which belongs to the P-ala subtype, is the most commonly studied EBNA1. However, the V-val subtype predominates in Taiwan. Thus, we cloned and expressed a 
A
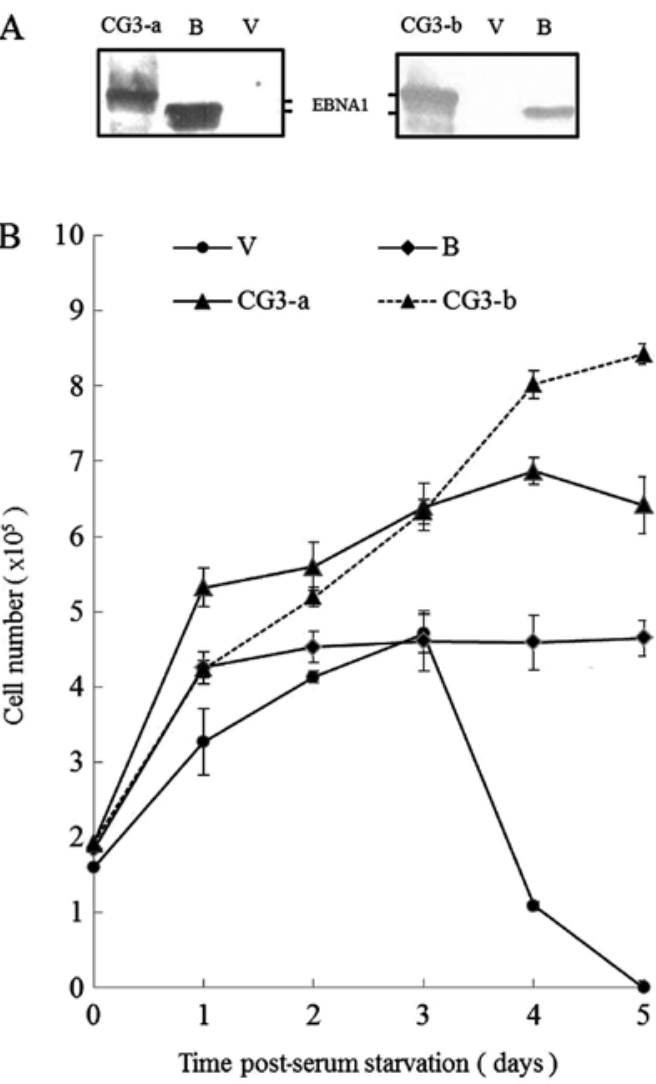

C
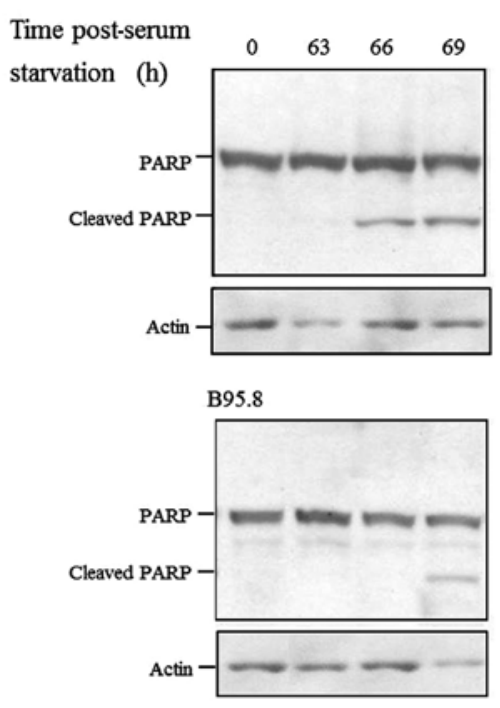

CG3

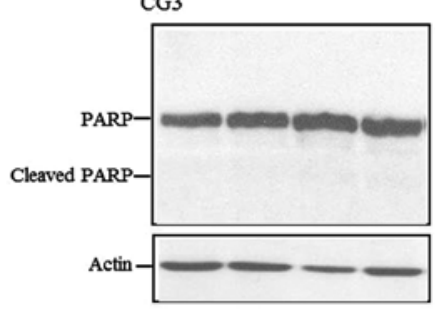

Figure 6. Effects of EBNA1 on cell survival under serum starvation. (A) The CG3 and B95.8 EBNA1s were stably expressed in 293 cells, as confirmed by western blotting. (B) Growth kinetics of 293 cells stably expressing the CG3 and B95.8 EBNA1s under serum-free conditions. Control cells (V) expressed the drug-resistant gene (vector) alone. V, B, CG3-a and CG3-b indicate 293 cells stably expressing the empty vector, B95.8 EBNA1, and two clones of CG3 EBNA1, respectively. (C) Immunoblot detection of PARP cleavage in lysates from control cells (V) and those expressing the B95.8 or CG3-a EBNA1s following serum starvation for 63,66 and $69 \mathrm{~h}$.

non-prototypic EBNA1 subtype from a CG3 cell line, an EBV-carrying lymphoblastoid cell line that was derived from a Taiwanese patient with juvenile chronic myeloid leukemia. The GC-rich middle region of the EBNA1-encoding gene, which encodes the glycine-alanine repeats, can complicate the PCR amplification of the EBNAl gene (28). To clone the full-length V-val EBNA1 gene from CG3 cells, we therefore used commercial resolution buffer to improve the efficiency of the PCR reaction. Our data indicate that we successfully PCR amplified the full-length EBNA1 gene from CG3 cells. Using our protocol, therefore, the full-length EBNA1 genes of various subtypes may be readily amplified from EBV-positive samples, potentially facilitating studies of their functional differences (especially in the context of EBV-related disease).

Variations in the EBNA1 sequences relative to the prototype strain, B95.8, were first described in EBV isolates from NPC samples obtained in Hong Kong (28). These variations were subsequently classified into the V-val subtype, based on the system established by Gutiérrez et al (30). In the present study, we demonstrate that the EBNA1 protein expressed in the CG3 cell line, which is associated with chronic myeloid leukemia, also belongs to the V-val subtype. This finding is consistent with previous studies that V-val EBNA1 is the predominant subtype detected in healthy Taiwanese individuals and Taiwanese patients with EBV-associated diseases $(32,37,47)$. Furthermore, our sequencing results revealed only minor changes in the EBNA1 sequence (one and two amino acid substitutions in the $\mathrm{N}$ - and C-terminal domains, respectively) relative to the EBNA1 sequences found in NPC samples from Hong Kong. In the future, larger-scale sequence analyses and functional analyses of samples collected from different tumors are required to elucidate the role of these sequence variations in the tissue tropism of EBV.

To further characterize EBNA1 and establish a good source of antigens and antibodies for use in functional studies and as diagnostic reagents, we subcloned the $\mathrm{N}$ - and $\mathrm{C}$-terminal regions of the CG3 V-val EBNA1 into a bacterial expression vector to produce His-tagged fusion proteins, and then raised the corresponding antibodies in rabbits. The purified EBNA1 fusion proteins and rabbit polyclonal antibodies constitute specific and biologically safe reagents. They are good reagents for the diagnosis of EBV infection, the immunodetection of EBNA1 in western blot analysis, and potentially for a largescale ELISA-based screening program that may provide diagnostic and epidemiological information on EBV-related diseases.

Most of the existing studies regarding the functional roles of EBNA1 in the tumorigenesis of EBV have used the cloned B95.8 EBNA1 (a P-ala EBNA1) or EBV-containing cell lines carrying EBNA1 of unknown subtypes. In the present study, we constructed a eukaryotic expression plasmid containing a V-val EBNA1 gene originally derived from chronic myeloid 
leukemia. We found that the expression of this V-val EBNA1 in 293 cells supported cell growth and inhibited apoptosis (two important characteristics for an oncogene) under serum starvation. This V-val EBNA1 expression plasmid provides a good experimental tool to determine, whether the V-val EBNA1 can promote oncogenesis in the absence of other EBV sequences, and whether the V-val EBNA1 and the P-ala prototype show functional differences in contributing to the development of EBV-associated malignancies.

Although NPC has been identified worldwide, its incidence varies among population groups, with the highest incidence observed in southern Chinese males (48). V-val EBNA1 is the only viral protein that has been observed in all NPC samples from southern China $(28,33,36,37,49)$. Thus, future studies should be conducted to examine how this EBNA1 subtype may contribute to the pathogenesis of NPC in high-incidence areas. Since we successfully established a protocol for amplifying the full-length EBNA1 gene from EBV-positive samples, it should now be possible to clone and express the V-val EBNA1 from various NPC samples. Previous studies have indicated that some of the variations observed between the P-ala and V-val EBNA1s lie in the DNA-binding region $(28,33,49)$. EBNA1 and FR derived from Asian isolates induced higher transcriptional activity than those from the B95.8 strain, as assessed using a luciferase reporter system $(33,35)$. Findings of a previous study have indicated that the expression of V-val EBNA1 isolated from an NPC biopsy had no effect on 293 cell growth under normal growth conditions (49). However, the authors observed that the fluorescence from GFP reporter was more intense in 293 cells expressing a V-val-EBNA1-GFP plasmid compared with a P-ala-EBNA1-GFP plasmid after long-term culture. $\mathrm{V}$-val and P-thr EBNA1s have been detected in NPC samples obtained from Beijing Hospital (31) and North Africa $(28,30)$, whereas P-ala and P-thr (but not V-val) were detected in NPC samples from Denmark, which is an area of low NPC risk (31). The V-leu subtype has not been found in NPC patients, even in areas in which it is not a rare EBNA 1 subtype $(30,46)$. Since NPC differs clinically and molecularly in different regions of the world (50), we conclude a possible association of V-val with undifferentiated NPC in southern China, and/or the possibility that yet-unidentified genetic polymorphisms in EBNA1 (e.g., the number of internal glycine-alanine repeats) may be important in the tumorigenesis of NPC.

The results of the present study suggest that V-val EBNA1 isolated from a chronic myeloid leukemia-derived cell line potentially contribute to regulating proliferation and antiapoptosis in 293 cells. We also introduced a cloning strategy that may be used to compare the biological functions of fulllength EBNA1s isolated from specific populations, such as patients with EBV-associated disease as compared to healthy individuals from the same geographic region. Such investigations are likely to considerably improve our understanding of the polymorphic patterns of EBNA1 and their associations with malignancies in different geographic regions.

\section{Acknowledgements}

This study was supported by the Chang Gung Memorial Hospital (CMRPD32039, 160071-3, and 190111-2).

\section{References}

1. Young LS and Murray PG: Epstein-Barr virus and oncogenesis: from latent genes to tumours. Oncogene 22: 5108-5121, 2003.

2. Schepers A, Ritzi M, Bousset K, et al: Human origin recognition complex binds to the region of the latent origin of DNA replication of Epstein-Barr virus. EMBO J 20: 4588-4602, 2001.

3. Sears J, Kolman J, Wahl GM and Aiyar A: Metaphase chromosome tethering is necessary for the DNA synthesis and maintenance of oriP plasmids but is insufficient for transcription activation by Epstein-Barr nuclear antigen 1. J Virol 77: 11767-11780, 2003.

4. Nanbo A, Sugden A and Sugden B: The coupling of synthesis and partitioning of EBV's plasmid replicon is revealed in live cells. EMBO J 26: 4252-4262, 2007.

5. Sugden B and Warren N: A promoter of Epstein-Barr virus that can function during latent infection can be transactivated by EBNA-1, a viral protein required for viral DNA replication during latent infection. J Virol 63: 2644-2649, 1989.

6. Altmann M, Pich D, Ruiss R, Wang J, Sugden B and Hammerschmidt W: Transcriptional activation by EBV nuclear antigen 1 is essential for the expression of EBV's transforming genes. Proc Natl Acad Sci USA 103: 14188-14193, 2006.

7. Gahn TA and Sugden B: An EBNA-1-dependent enhancer acts from a distance of 10 kilobase pairs to increase expression of the Epstein-Barr virus LMP gene. J Virol 69: 2633-2636, 1995.

8. Sample J, Henson EB and Sample C: The Epstein-Barr virus nuclear protein 1 promoter active in type I latency is autoregulated. J Virol 66: 4654-4661, 1992.

9. Sung NS, Wilson J, Davenport M, Sista ND and Pagano JS: Reciprocal regulation of the Epstein-Barr virus BamHI-F promoter by EBNA-1 and an E2F transcription factor. Mol Cell Biol 14: 7144-7152, 1994.

10. Frappier L: Role of EBNA1 in NPC tumourigenesis. Semin Cancer Biol 22: 154-161, 2012.

11. Westhoff Smith D and Sugden B: Potential cellular functions of Epstein-Barr nuclear antigen 1 (EBNA1) of Epstein-Barr virus. Viruses 5: 226-240, 2013.

12. Kennedy G, Komano J and Sugden B: Epstein-Barr virus provides a survival factor to Burkitt's lymphomas. Proc Natl Acad Sci USA 100: 14269-14274, 2003.

13. Holowaty $\mathrm{MN}$, Zeghouf $\mathrm{M}, \mathrm{Wu} \mathrm{H}$, et al: Protein profiling with Epstein-Barr nuclear antigen-1 reveals an interaction with the herpesvirus-associated ubiquitin-specific protease HAUSP/ USP7. J Biol Chem 278: 29987-29994, 2003.

14. Holowaty MN, Sheng Y, Nguyen T, Arrowsmith C and Frappier L: Protein interaction domains of the ubiquitin-specific protease, USP7/HAUSP. J Biol Chem 278: 47753-47761, 2003.

15. Wilson JB, Bell JL and Levine AJ: Expression of Epstein-Barr virus nuclear antigen-1 induces B cell neoplasia in transgenic mice. EMBO J 15: 3117-3126, 1996.

16. Murakami M, Lan K, Subramanian C and Robertson ES: Epstein-Barr virus nuclear antigen 1 interacts with $\mathrm{Nm} 23-\mathrm{H} 1$ in lymphoblastoid cell lines and inhibits its ability to suppress cell migration. J Virol 79: 1559-1568, 2005.

17. Kaul R, Murakami M, Choudhuri $\mathrm{T}$ and Robertson ES: Epstein-Barr virus latent nuclear antigens can induce metastasis in a nude mouse model. J Virol 81: 10352-10361, 2007.

18. O'Neil JD, Owen TJ, Wood VH, et al: Epstein-Barr virus-encoded EBNA1 modulates the AP-1 transcription factor pathway in nasopharyngeal carcinoma cells and enhances angiogenesis in vitro. J Gen Virol 89: 2833-2842, 2008.

19. Wood VH, O'Neil JD, Wei W, Stewart SE, Dawson CW and Young LS: Epstein-Barr virus-encoded EBNA1 regulates cellular gene transcription and modulates the STAT1 and TGFbeta signaling pathways. Oncogene 26: 4135-4147, 2007.

20. Flavell JR, Baumforth KR, Wood VH, et al: Down-regulation of the TGF-beta target gene, PTPRK, by the Epstein-Barr virus encoded EBNA1 contributes to the growth and survival of Hodgkin lymphoma cells. Blood 111: 292-301, 2008.

21. Valentine R, Dawson $\mathrm{CW}, \mathrm{Hu} \mathrm{C}$, et al: Epstein-Barr virusencoded EBNA1 inhibits the canonical NF-kappaB pathway in carcinoma cells by inhibiting IKK phosphorylation. Mol Cancer 9: 1, 2010.

22. Gruhne B, Sompallae R, Marescotti D, Kamranvar SA, Gastaldello S and Masucci MG: The Epstein-Barr virus nuclear antigen-1 promotes genomic instability via induction of reactive oxygen species. Proc Natl Acad Sci USA 106: 2313-2318, 2009. 
23. Gruhne B, Sompallae R and Masucci MG: Three Epstein-Barr virus latency proteins independently promote genomic instability by inducing DNA damage, inhibiting DNA repair and inactivating cell cycle checkpoints. Oncogene 28: 3997-4008, 2009.

24. Hennessy $\mathrm{K}$ and Kieff E: One of two Epstein-Barr virus nuclear antigens contains a glycine-alanine copolymer domain. Proc Natl Acad Sci USA 80: 5665-5669, 1983.

25. Hennessy K, Heller M, van Santen V and Kieff E: Simple repeat array in Epstein-Barr virus DNA encodes part of the Epstein-Barr nuclear antigen. Science 220: 1396-1398, 1983.

26. Allday MJ and MacGillivray AJ: Epstein-Barr virus nuclear antigen (EBNA): size polymorphism of EBNA 1. J Gen Virol 66: 1595-1600, 1985

27. Levitskaya J, Coram M, Levitsky V, et al: Inhibition of antigen processing by the internal repeat region of the Epstein-Barr virus nuclear antigen-1. Nature 375: 685-688, 1995.

28. Snudden DK, Smith PR, Lai D, Ng MH and Griffin BE: Alterations in the structure of the EBV nuclear antigen, EBNA1, in epithelial cell tumours. Oncogene 10: 1545-1552, 1995.

29. Chang CM, Yu KJ, Mbulaiteye SM, Hildesheim A and Bhatia K The extent of genetic diversity of Epstein-Barr virus and its geographic and disease patterns: a need for reappraisal. Virus Res 143: 209-221, 2009.

30. Gutiérrez MI, Raj A, Spangler G, et al: Sequence variations in EBNA-1 may dictate restriction of tissue distribution of Epstein-Barr virus in normal and tumour cells. J Gen Virol 78: $1663-1670,1997$

31. Sandvej K, Zhou XG and Hamilton-Dutoit S: EBNA-1 sequence variation in Danish and Chinese EBV-associated tumours: evidence for geographical polymorphism but not for tumourspecific subtype restriction. J Pathol 191: 127-131, 2000.

32. Wang JT, Sheeng TS, Su IJ, Chen JY and Chen MR: EBNA-1 sequence variations reflect active EBV replication and disease status or quiescent latency in lymphocytes. J Med Virol 69: 417-425, 2003

33. Do NV, Ingemar E, Phi PT, et al: A major EBNA1 variant from Asian EBV isolates shows enhanced transcriptional activity compared to prototype B95.8. Virus Res 132: 15-24, 2008.

34. Habeshaw G, Yao QY, Bell AI, Morton D and Rickinson AB: Epstein-Barr virus nuclear antigen 1 sequences in endemic and sporadic Burkitt's lymphoma reflect virus strains prevalent in different geographic areas. J Virol 73: 965-975, 1999.

35. Mai SJ, Xie D, Huang YF, et al: The enhanced transcriptional activity of the V-val subtype of Epstein-Barr virus nuclear antigen 1 in epithelial cell lines. Oncol Rep 23: 1417-1424, 2010.

36. Zhang XS, Wang HH, Hu LF, et al: V-val subtype of Epstein-Barr virus nuclear antigen 1 preferentially exists in biopsies of nasopharyngeal carcinoma. Cancer Lett 211: 11-18, 2004.
37. Wang WY, Chien YC, Jan JS, Chueh CM and Lin JC: Consistent sequence variation of Epstein-Barr virus nuclear antigen 1 in primary tumor and peripheral blood cells of patients with nasopharyngeal carcinoma. Clin Cancer Res 8: 2586-2590, 2002.

38. Chang YS, Shih LY, Peng CL, Chen SH and Liu ST: Characterization of two newly established EBV-containing lymphoblastoid cell lines from patients with myeloid leukemias. Leuk Res 14: 309-320, 1990.

39. Miller G, Shope T, Lisco H, Stitt D and Lipman M: Epstein-Barr virus: transformation, cytopathic changes, and viral antigens in squirrel monkey and marmoset leukocytes. Proc Natl Acad Sci USA 69: 383-387, 1972.

40. Pulvertaft JV: A study of malignant tumours in Nigeria by shortterm tissue culture. J Clin Pathol 18: 261-273, 1965.

41. Hinuma Y and Grace JT Jr: Cloning of immunoglobulinproducing human leukemic and lymphoma cells in long-term cultures. Proc Soc Exp Biol Med 124: 107-111, 1967.

42. Arrand JR, Walsh-Arrand JE and Rymo L: Cytoplasmic RNA from normal and malignant human cells shows homology to the DNAs of Epstein-Barr virus and human adenoviruses. EMBO J 2: 1673-1683, 1983.

43. Graham FL, Smiley J, Russell WC and Nairn R: Characteristics of a human cell line transformed by DNA from human adenovirus type 5. J Gen Virol 36: 59-74, 1977.

44. Baer R, Bankier AT, Biggin MD, et al: DNA sequence and expression of the B95-8 Epstein-Barr virus genome. Nature 310: 207-211, 1984.

45. Wang TC and Chao M: Molecular cloning and expression of the hepatitis delta virus genotype IIb genome. Biochem Biophys Res Commun 303: 357-363, 2003.

46. Bhatia K, Raj A, Guitierrez MI, et al: Variation in the sequence of Epstein-Barr virus nuclear antigen 1 in normal peripheral blood lymphocytes and in Burkitt's lymphomas. Oncogene 13: 177-181, 1996.

47. Chen MR, Tsai CH, Wu FF, Kan SH, Yang CS and Chen JY: The major immunogenic epitopes of Epstein-Barr virus (EBV) nuclear antigen 1 are encoded by sequence domains which vary among nasopharyngeal carcinoma biopsies and EBV-associated cell lines. J Gen Virol 80: 447-455, 1999.

48. Niedobitek G: Epstein-Barr virus infection in the pathogenesis of nasopharyngeal carcinoma. Mol Pathol 53: 248-254, 2000.

49. Mai SJ, Ooka T, Li DJ, et al: Functional advantage of NPC-related V-val subtype of Epstein-Barr virus nuclear antigen 1 compared with prototype in epithelial cell line. Oncol Rep 17: 141-146, 2007.

50. de Thé G: Nasopharyngeal carcinoma. In: Viral Infections of Humans, Epidemiology and Control. Evans AS and Kazslow RA (eds). 4th edition. Plenum Medical Book Co, New York, NY, pp935-967, 1997. 